able simplification of procedure. Universities and their colleges were excluded from the operation of the Bill, and the segregation of the great schools of the I868 Act was to be continued by their reservation from the more sweeping clauses; indeed, Eton and Winchester would only be affected so far as their governing bodies consented. Safeguards relating to the religious character of certain foundations were introduced, although Clause 16 states, and very rightly, that "in making a scheme regard" shall be had primarily to the educational advantages to be derived from the scheme." May we not hope that the matter will be discussed and legislated upon in this spirit?

\section{G. F. Daniell.}

\section{UNIVERSITY AND EDUCATIONAL INTE LLIGENCE.}

Cambridge.- The next combined examination for sixtysix entrance scholarships and various exhibitions at Pembroke, Gronville and Caius, King's, Jesus, Christ's, St John's, and Emmanuel Colleges will be held on Tuesday, December 3, and following days. Mathematics, classics, and natural sciences will be the subjects of examination at all the above-mentioned colleges. A candidate for a scholarship or exhibition at any of the seven colleges must not be more than nineteen years of age on October I, 1907 . Forms of application must be sent in on or before Tuesday, November 26.

Mr. A. H. Lees, of King's College, has been appointed to the studentship in medical entomology for the period of one year. The studentship was recently established on the basis of a grant from the "Tropical Diseases Research Fund," administered by the Colonial Office. Mr. Lees will pursue research und the flirection of the Quick professor of bighogy.

Of the four fellowship awarded last week at Trinity College, two were for cassics; one of the remaining two was awarded t $\mathrm{Mr}$. A. S.. Eddington, senior wrangler in 1904, and fyst clasf first division, part ii. of the mathematical trifos 905 , and the other to $\mathrm{Mr}$. V. H. Mottran, first clas a a r i. of the natural sciences tripos, I903, and firsy tiass (physiology) in the same tripos, part ii., 1905 .

$\mathrm{Mr}$ A. Wood, who took his degree in chemistry and physits in 1904 as an advanced student, has been elected to a fellowship at Emmanuel College.

Mr. A. Bery, of King's College, has been appointed chairman of exwiners for the mathematical tripos, part i. (old regulatiohs), igo8.

At a Congfegation to be held to-day, the honorary degree of Doctor of Science will be conferred on Prof. Emil Fischer, of Berlin.

Liverpool.-Prof. J. Reynolds Green, F.R.S., lately professor of botany to the Pharmaceutical Society of Great pritain, pys been opted the Hartley lectureship in departmegt thyough Aintree

Dr. A. Arindley has been appointed principal of the Goverhment School of Engineering at Ghizeh, Cairo. The school is under the Egyptian Ministry of Education for the training of engineers for the various departments of the Egyptian public works.

Mr. W. MacGregor Wallace has been elected lecturer on applied mechanics in place of Dr. Grindley (resigned).

Manchester.--The honorary degree of Ph.D. has been conferred upon Prof. Ernest Rutherford by the University of Giessen.

The following appointments 1002 been made :-Dr. C. H. Weizmann, leftyer in hemistry; Mr. J. N. Pring, demonstrato in elecemistry; Mr. F. H. Gravely, assistant lecfgrer afdudemonstrator in zoology. Mr. Frank physiology on his appointment to a similar post at the Armstrong College.

Dr. R. S. Hutton, on leaving Manchester, is resigning his position as lecturer in electrochemistry and assistant director of the physical laboratories, but has been appointed a special leciurer in electrochemistry.

No. I $98 \mathrm{I}$, VOL. 76]
THE Herter lectoses before the medical department of the Johns. Hopking University are to be given this session by Prof. E. A. Zchäfer, F.R.S., professor of physiology in the University Edinburgh, at the end of April, 1908.

We have received the current issue of the year-book of the Michigan College of Yines. It covers 136 pages, and contains fyet details of the courses arranged for the session 1907. The coy eshare admirably planned, and the situatio collyge in the copper- and iron-ore district of Milhtgan, where its students live in a mining atmosphere, has brought to the institution a large measure of success.

UNDER the will of the late Dr. Nathaniel Rogers, the Senate of the University of London offers a prize of rool., open for competition to all members of the medical profession in the United Kingdom, for the best essay or dissertation setting forth the results of original investigations made bf the candidate on any medical pathological subject (uving the preceding two years. Candidates will be permitted to present papers published during the preceding year af the dissertation. The essay or dissertation, by preference typewritten or printed, must be sent in not later than May I, I908, addressed to the clerk of committees at the University.

THE prospectus for the session 1907-8 of the Belfast Municipal Technical Institute should prove of real assistance to intending students seeking guidance in planning their courses of work. It is quite clear from the volume, which runs to nearly 250 pages, that the chief object of the institute is to proyide instruction in the principles of those arts and.scieyces which bear directly or indirectly upon the trades and industries of Belfast, and to show by experiment be w these principles may be applied to secure industrial atvancement. The classes are designed to assist persons engaged during the day in handicrafts or business, and desire to supplement the knowledge gained in the workshop or warehouse. The time-table of classes is published as a separate pamphlet, and with it a sensible letter of advice to students from the principal of the institute, Mr. Fras. C. Forth, indicating several directions in which students can assist the staff to secure success in the work of the various departments. Arrangements have been made for full courses of study in the various branches of science, art, technology, and commercial subjects.

AmoNG the advanced lectures on scientific subjects announced in connection with the University of London to be held during the present session may be mentioned a course of eight lectures, by Mr. A. D. Hall, on the function of the mineral constituents of the soil in the nutrition of plants, $\rightarrow$ be given at the Chelsea Physic Garden on dates to be announced later. Mr. J. B. Leathes commended a course of eight lectures on October I $_{5}$ at University physiological laboratory on problems in anithal metabolism. At the same place four lectures on the construction of diets in health will be commenced by Dr. E. I. Spriggs on November 8 . Three lectures on the principles of classification will be given at University College by Mr. G. A. Boulenger, F.R.S., beginning on October 28 , and three lectures by Mr. J. T. Cunningham on sexual dimorphism, beginning on November 18 . The University reader in meteorology will deliver at the University a course of twelve lectures on meteorological organisation and methods of dealing with meteorological observation, commencing on October 21.

THE Electrician for October. II contains an interesting description of the electrotechnic institute of the Technical University in Kaylsruhe Baden, by Mr. Stanley P. Smith.

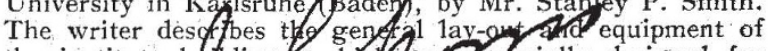
the institute blyding, which for specially designed for the various branch of frgineering carried on within them, but the desciption mainly deals with the electrotechnic institut itself. The cost and equipment of this building was between $2700 \mathrm{l}$. and $2800 \mathrm{l}$, and from the plans and description given there is no doubt that it is very perfectly arranged and fitted up. The general idea of the 
curriculum followed is also given, and it will be of interest to both engineers and others to note that the course at Karlsruhe includes political economy and labour problems. There is also a greater tendency in Germany for the various branches of engineering to be treated by professors who are also engaged in practical work. On another point the institution at Karlsruhe differs from the colleges in this country, namely, the students themselves. There appear to be no student organisations, and the sociability so characteristic of an English college is almost entirely wanting.

\section{SOCIETIES AND ACADEMIES.}

\section{LONDON.}

Royal Society, June 27.- "The Annealing of Copper, with Special Reference to Dilatation." By Prof. T. Turner and D. Mi Levy. Communicated by Prof. J. H. Poynting, F.R.S.

The authors have employed a special form of extensowheter, in conjunction with a Le Chatelier pyrometer, in order to trace the changes in the length of metallic rods during the process of annealing and have thus obtained continuous curves connecting dilatation and temperature. The rods, which were $\frac{1}{4}$-inch. in square section and 35 inches long, were uniformly and regularly heated in a gas-fired furnace. The chief feature of the apparatus was the use of water-cooled copper tubes for connecting the rod with the extensometer, a plug of non-conducting material being used to prevent any cooling of the end of the rod by the water. By this method the whole of the rod was in the furnace and uniformly heated, while the rest of the system was maintained at a constant temperature. The water-cooled tube attached to one end of the rod was firmly clamped, the other being free to move. On to this tube was screwed a brightly polished brass disc, against which pressed a finely rounded projection attached to the short arm of a bell-crank lever, which, traversing a scale, indicated the expansion of the rod.

The scale was divided into millimetres, each millimetre representing $\mathrm{I} / \mathrm{I} 200-\mathrm{inch}$ expansion, corresponding to a magnification of $48: \mathrm{r}$. The suitability and delicacy of the apparatus were ascertained by testing bars of wrought iron and steel; from the former a regular line was obtained, while the latter gave a curve showing a marked change of volume at the critical point (about $690^{\circ} \mathrm{C}$.), thus agreeing with Le Chatelier's results obtained by an entirely different method. Hard-drawn copper bars gave a perfectly regular line, similar to that obtained with wrought iron; annealed copper also gave a straight line. Experiments on rods of brass of different composition, on gun-metal, and on phosphor bronze gave similar results.

It thus appears that the change from the hard, elastic condition of worked copper and copper alloys to that of extremely soft metal is not accompanied by any alteration in length. On the other hand, it is known that allotropic changes in an element, such as occur in pure iron at about $880^{\circ}$, or such chemical constitutional changes as occur in iron-carbon alloys at critical temperatures, are accompanied by marked alterations in volume, and the authors therefore conclude that the changes brought about by mechanical work, or by annealing of worked metals, produce only internal re-arrangement of the metallic molecules, but are of a different order from the chemical and physical changes, such as are correctly regarded as allotropic.

"Experiments on a New Kathode Dark Space in Helium and Hydrogen." By F. W. Aston. Communicated ty Prof. J. H. Poynting, F.R.S.

This paper is a description of a new dark space, close up to the kathode and inside the Crookes dark space, discovered by the author while investigating the length of the latter phenomenon in helium, and later found to be exhibited in a less marked degree in hydrogen. The length of the new dark space, which under measurable conditions varies from $0.2 \mathrm{~mm}$. to $x \cdot 0 \mathrm{~mm}$., is almost unalfected by the pressure of the gas, but varies roughly with the inverse square root of the current density.

Careful observations show that the fall of potential across the new dark space is constant for the same gas under NO. I 98 I, VOL. 76 ] all observed conditions, and is in helium 30 volts, in hydrogen $\mathrm{r}_{5}$ volts. The phenomenon may be accounted for by the supposition that the energy required to ionise a molecule of helium is a definite quantity, and that an electron liberated from the surface of the kathode virtually at rest must fall freely through a definite potential in order to acquire that energy, so that the new dark space may be regarded as the distance through which the electrons fall in order to attain sufficient energy to ionise the gas by collision with its molecules.

The intense blackness of the new dark space in pure helium bears out this theory, by which also the following effect was predicted:- Since the behaviour of electrons liberated from molecules of the gas by collisions should be the same as that of those derived from the kathode, if the ionisation just beyond a potential distance from the kathode of 30 volts is sufficiently concentrated there will be a further maximum of ionisation-and therefore of light-just beyond a potential distance of 60 volts, another beyond 90 volts, and so on, each getting less definite than the previous one, so that the light in the Crookes dark space should be striated in appearance. By suitable adjustment of conditions, several successive striations can be seen and photographed in helium. Combining the potential differences obtained from the length of the new dark space with the accepted values of $e$ and $m$ for the electron the following values of the energy required to ionise and the velocity of the ionising electron are obtained :--

Hydrogen $\quad \ldots \quad .1 .7 \times 10^{-11}$ ergs $2.25 \times 10^{8} \mathrm{~cm}$. per sec.

Helium... ... $3.4 \times 10^{-11}, 3^{2} 2 \times 10^{8} \quad$,

Not the slightest indication of the phenomenon has jet been observed in any other gas.

Received July 5 "The Dispersion of Double Refraction in Relatien to Crystal Structure." By Dr. T. H. Havelock Communicated by Prof. J. Larmor, Sec.R.S.

In this paper the object is to consider to what extent it is possible to regard double refraction, whether produced artificially or occurring in natural crystals, as due simply to an æolotropic distribution of similar particles. The ordinary theory of double refraction amounts to considering the medium as a collection of crystal molecules in cubical order, all the varieties of dispersion being postulated of the single particle; and similarly in artificial double refraction, the xolotropy is assumed to originate wholly in the individual molecule, and to be effected through the quasi-elastic force under which the polarisation electrons are supposed to vibrate. On the other hand, a theory which confines" the effect to a re-arrangement of the molecules in space will express the result by a modification of the effective electric force operative at each particle. From this point of view the author develops a theory of the optical properties of a homogeneous assemblage of isotropic particles. If the medium behaves like a uniaxal crystal, it is found that the double refraction, equal to the difference between the two principal indices, is proportional to $\left(n_{0}{ }^{2}-I\right)^{2} / n_{0}$, where $n_{0}$ is a mean value measuring the refractive index of a medium composed of the same particles in the same density, but arranged in simple cubical order.

This gives a law of dispersion of the double refraction, provided the dispersion of the mean index $n_{0}$ is known. The formula is applied first to the double refraction produced artificially in simple isotropic media. Experimenta data are available for the dispersion of the double refraction produced in carbon disulphide by an electric field, and these results are found to agree well with the formula given above.

In the case of natural crystals, where the effect is larger, the theory indicates that the quantity

$$
\left(n_{1}{ }^{2}-\mathrm{r}\right)^{-1}-\left(n_{2}{ }^{2}-\mathrm{r}\right)^{-1}
$$

should be independent of the wave-length, $n_{1}$ and $n$, being the ordinary and extraordinary indices of the crystal. This relation is found to hold very well for quartz over a wide range, and gives in this case the equation

$$
n_{2}{ }^{2}-n_{1}{ }^{2}=0.0144 \mathrm{I}\left(n_{1}{ }^{2}-\mathrm{I}\right)\left(n_{2}{ }^{2}-\mathrm{I}\right) \text {. }
$$

It follows, further, that the double refraction decreases in absolute value with the mean index $n_{0}$, that is, it decreases in general with increasing wave-length. This is the rule of dispersion in most actual erystals, but there 